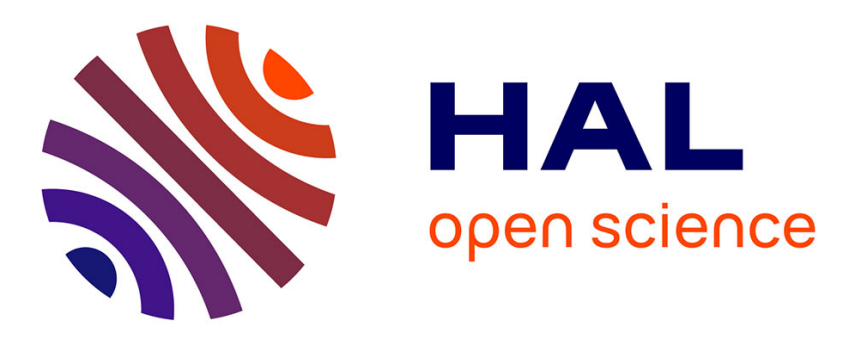

\title{
Analysis of Sound Signals with High Resolution Matching Pursuit
}

Rémi Gribonval, Emmanuel Bacry, Stéphane Mallat, Philippe Depalle, Xavier Rodet

\section{- To cite this version:}

Rémi Gribonval, Emmanuel Bacry, Stéphane Mallat, Philippe Depalle, Xavier Rodet. Analysis of Sound Signals with High Resolution Matching Pursuit. Proc. IEEE Symp. Time-Freq. and TimeScale Anal. (TFTS'96), Jun 1996, Paris, France. pp.125-128, 10.1109/TFSA.1996.546702 . inria00576196

\section{HAL Id: inria-00576196 \\ https://hal.inria.fr/inria-00576196}

Submitted on 13 Mar 2011

HAL is a multi-disciplinary open access archive for the deposit and dissemination of scientific research documents, whether they are published or not. The documents may come from teaching and research institutions in France or abroad, or from public or private research centers.
L'archive ouverte pluridisciplinaire HAL, est destinée au dépôt et à la diffusion de documents scientifiques de niveau recherche, publiés ou non, émanant des établissements d'enseignement et de recherche français ou étrangers, des laboratoires publics ou privés. 


\title{
ANALYSIS OF SOUND SIGNALS WITH HIGH RESOLUTION MATCHING PURSUIT
}

\author{
R. Gribonval E. Bacry S. Mallat ${ }^{\dagger}$ Ph. Depalle and X. Rodet ${ }^{\ddagger}$ \\ IRCAM, 1 place Igor-Stravinsky, 75004 PARIS, FRANCE. \\ e-mail : gribonva@clipper.ens.fr, fax : (33)-1-42772947
}

\begin{abstract}
Sound recordings include transients and sustained parts. Their analysis with a basis expansion is not rich enough to represent efficiently all such components. Pursuit algorithms choose the decomposition vectors depending upon the signal properties. The dictionary among which these vectors are selected is much larger than a basis. Matching Pursuit is fast to compute, but can provide coarse representations. Basis Pursuit gives a better representation but is very expensive in terms of calculation time. This paper develops a High Resolution Matching Pursuit : it is a fast, high time-resolution, time-frequency analysis algorithm, that makes it likely to be used for musical applications.
\end{abstract}

\section{INTRODUCTION}

The complexity of structures encountered in a sound recording requires to develop adaptive lowlevel representations. Although the decomposition of such a signal in a basis entirely characterizes it $\Gamma$ this basis is a minimal set of vectors that is not rich enough to represent efficiently all components. Some signal structures are diffused across many basis elements and are thus difficult to analyze from this expansion.

Indeed $\Gamma$ sound recordings include transients that are well represented by short waveformsTand sustained parts that are more efficiently decomposed over long waveforms with short frequency

\footnotetext{
${ }^{*}$ CNRS Paris 7

${ }^{\dagger} \mathrm{CMAP}$ Ecole Polytechnique

¡IRCAM
}

support. Pursuit algorithms choose the decomposition vectors depending upon the signal properties. These vectors are selected among a family of waveforms that is much larger than a basis which is called a dictionary.

The High Resolution Matching Pursuit (HRMP) developed in this paper is a fast algorithm providing high time-resolution time-frequency representations 5 that enables it to be used for musical applications.

\section{MATCHING PURSUIT}

A dictionary is a family of vectors $\mathcal{D}=\left(g_{\gamma}\right)_{\gamma \in \Gamma}$ included in a Hilbert space $H \Gamma$ with a unit norm $\left\|g_{\gamma}\right\|=1$. A matching pursuit is an iterative algorithm that decomposes the signal over dictionary vectors as follows.

Let $R^{0} f=f$. We suppose that we have computed the $n^{\text {th }}$ order residue $R^{n} f \Gamma$ for $n \geq 0$. We then choose an element $g_{\gamma_{n}} \in \mathcal{D}$ which "closely" matches the residue $R^{n} f$ Tin the sense that

$$
\left|C\left(R^{n} f, g_{\gamma_{n}}\right)\right|=\sup _{\gamma \in \Gamma}\left|C\left(R^{n} f, g_{\gamma}\right)\right|,
$$

where $C\left(f, g_{\gamma}\right)$ is a correlation function that measures the similarity between $f$ and $g_{\gamma}$.

The residue $R^{n} f$ is then sub-decomposed into

$$
R^{n} f=C\left(R^{n} f, g_{\gamma_{n}}\right) g_{\gamma_{n}}+R^{n+1} f,
$$

which defines the residue at the order $n+1$. In the Matching Pursuit (MP) Tinitially introduced by Mallat and Zhang [1] Tthe correlation function that is used is $C\left(f, g_{\gamma}\right)=<f, g_{\gamma}>$. The error $\left\|R^{n} f\right\|$ is then proved to decay to zero. Thus by 
iterating Eq. (2) we obtain

$$
f=\sum_{n=0}^{+\infty} C\left(R^{n} f, g_{\gamma_{n}}\right) g_{\gamma_{n}} .
$$

The structure of MP enables it to be implemented with a fast algorithm.

\section{GABOR DICTIONARY}

To analyze time and frequency localization properties of one-dimensional signals $\Gamma$ such as speech or music recordings $\Gamma$ we use a large dictionary of time-frequency atoms.

Let $g(t)=\frac{1}{\sqrt{2 \pi \sigma}} \exp \left(-\frac{t^{2}}{2 \sigma^{2}}\right)$ be a Gaussian function of unit norm. For any scale $s>0 \Gamma \bmod -$ ulation frequency $\xi$ and translation $u$ Twe denote $\gamma=(s, u, \xi)$ and define

$$
g_{\gamma}(t)=\frac{1}{\sqrt{s}} g\left(\frac{t-u}{s}\right) e^{i \xi t} .
$$

The index $\gamma$ is an element of the set $\Gamma=R^{+} \times R^{2}$. The function $g_{\gamma}(t)$ is centered at the abscissa $u$ and its energy is concentrated in a neighborhood of $u \Gamma$ whose size is proportional to $s$. Its Fourier transform is centered at the frequency $\omega=\xi$ Tand its energy is concentrated in a neighborhood of $\xi \Gamma$ whose size is proportional to $1 / s$. The timefrequency energy distribution of $f(t)$ is then defined by

$$
E f(t, \omega)=\sum_{n=0}^{+\infty}\left|C\left(R^{n} f, g_{\gamma_{n}}\right)\right|^{2} W g_{\gamma_{n}}(t, \omega) .
$$

where $W g_{\gamma_{n}}(t, \omega)$ is the Wigner distribution of $g_{\gamma_{n}} \Gamma$ i.e. a two-dimensional Gaussian "blob" in the time-frequency plane. Figure 1 and 2 display such time-frequency energy distributions.

\section{HIGH RESOLUTION MATCHING PURSUIT}

The Matching Pursuit is a greedy algorithm in that it optimizes at each step the amount of the signal energy it grasps. This often leads to a choice of features which globally fits the signal structures but is not best adapted to its local structures. Indeed $\Gamma$ for instance $\Gamma$ a signal composed of two bumps modulated by a sinusoidal wave at frequency $\xi$ (Figure 1-a) is first decomposed into a large atom at frequency $\xi$ (middle horizontal line on Figure 1-a-MP) that covers the time support of both bumps. Then Tin order to remove the energy created between the two bumps by this first atom $\Gamma M P$ chooses two atoms of the same size as the first one with frequencies $\xi+\Delta \xi$ (upper line) and $\xi-\Delta \xi$ (lower line).

Aiming at avoiding this problem DDonoho and Chen [2] introduced the Basis PursuitTwhich makes a full optimization $\Gamma$ by minimizing $\sum_{\gamma \in \Gamma}\left|\alpha_{\gamma}\right|$ over all possible decompositions $f=\sum_{\gamma \in \Gamma} \alpha_{\gamma} g_{\gamma}$. However this leads to large scale linear-programming problems and therefore is very expensive in terms of calculation time.

The new algorithm Tthat we called High Resolution Matching Pursuit (HRMP) Tis an enhanced version of Matching Pursuit (MP) Textending to time-frequency dictionaries the pursuit over nonmodulated spline dictionaries introduced by Jaggi et. al. [3]. It uses a different correlation function $\Gamma$ that allows the pursuit to emphasize local fit over global fit at each step. The fast algorithm structure of MP is however kept.

For each time-frequency atom $g_{\gamma}$ a set $I_{\gamma}$ of sub-atom indexes is introduced. $I_{\gamma}$ corresponds to smaller atoms $g_{\gamma_{i}}, \gamma_{i} \in I_{\gamma}$ with a time support included in the support of $g_{\gamma}$ Tand modulated at the same frequency. Let suppose that the atom $g_{\gamma}$ is chosen in a pursuit. $R f=f-C\left(f, g_{\gamma}\right) g_{\gamma}$ becomes the residue of this pursuit on the signal $f$. For all $\gamma_{i} \in I_{\gamma} \Gamma<R f, g_{\gamma_{i}}>$ represents the amount of "energy" of $R f$ located on the time-frequency support of $g_{\gamma_{i}}$. This amount must be smaller than the signal "energy" $<f, g_{\gamma_{i}}>$ at the same location. Moreover the corresponding decrease $<C\left(f, g_{\gamma}\right) g_{\gamma}, g_{\gamma_{i}}>$ of signal energy cannot be greater than the initial signal energy itself. This is formalized in Equations 6 and 7 :

$$
\begin{gathered}
\left|<R f, g_{\gamma_{i}}>\right| \leq\left|<f, g_{\gamma_{i}}>\right|, \\
\left|<C\left(f, g_{\gamma}\right) g_{\gamma}, g_{\gamma_{i}}>\right| \leq\left|<f, g_{\gamma_{i}}>\right| .
\end{gathered}
$$

From these relations we derive the new correlation function $C\left(f, g_{\gamma}\right)$ Wwhich maximizes the amount of signal energy that the pursuit can grasp $\Gamma$ when 


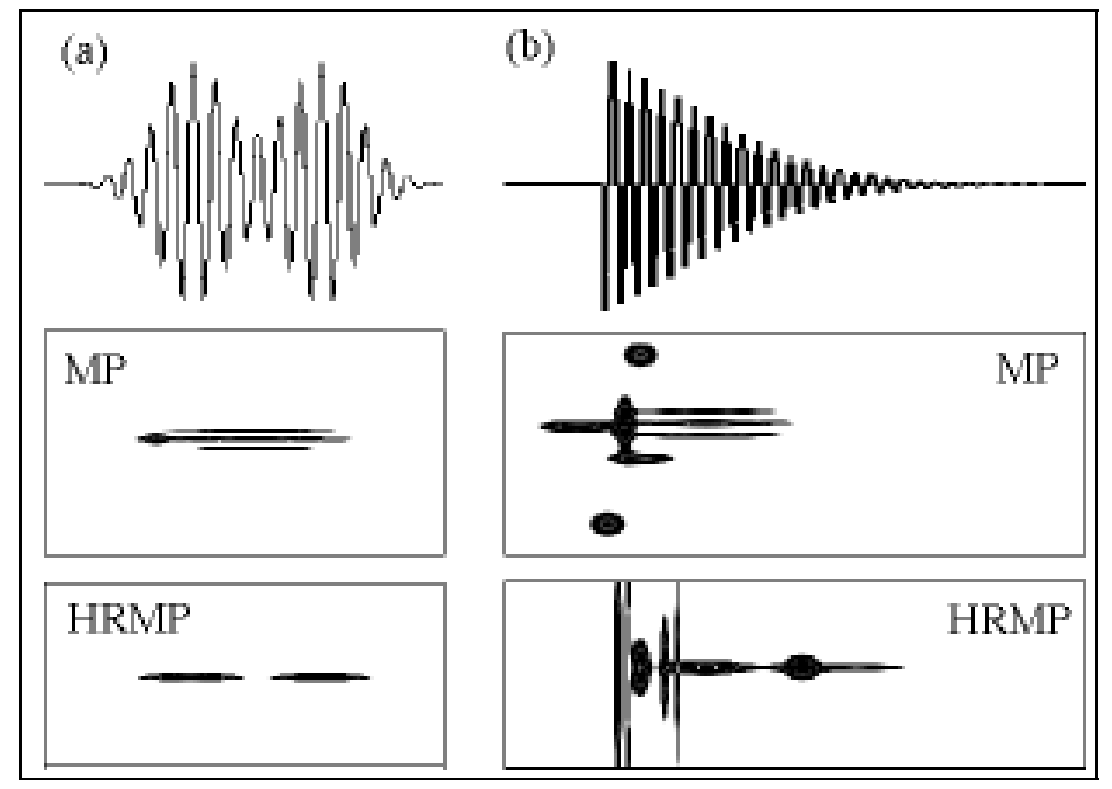

Figure 1: Time-Frequency distributions of signals (top) obtained with MP (middle) and HRMP (bottom): (a) two close bumpsTwith a four atom decomposition

(b) an attack pattern $\Gamma$ with a ten atom decomposition

choosing the atom $g_{\gamma}$ :

$$
C\left(f, g_{\gamma}\right)=\varepsilon \min _{\gamma_{i} \in I_{\gamma}} \frac{\left|<f, g_{\gamma_{i}}\right\rangle \mid}{\left|<g_{\gamma}, g_{\gamma_{i}}\right\rangle \mid}
$$

where $\varepsilon$ is evaluated as follows:

- if $<f, g_{\gamma_{i}}>$ have the same $\operatorname{sign} \Gamma$ for all $\gamma_{i} \in I_{\gamma}$ Tthen $\varepsilon$ is this common sign.

- else $\varepsilon=0$.

In MPT the inner-product $\Gamma$ used as a correlation function between a time-frequency atom and an audio signal disregards whether the signal contains energy on the whole time-frequency support of the chosen atom. On the contrary the new correlation function avoids creating energy at time locations where there was none. It can thus distinguish close time features as shown in Figure 1a-HRMP. Moreover it can avoid pre-echo effects $\Gamma$ i.e. creation of energy just before the beginning of the sound. Indeed Tas shown in Figure 1-bГMP introduces a pre-echo effect by choosing atoms that overlap the attack time-location $\Gamma$ whereas HRMP does not choose any such atom.
Figure 2 displays the time-frequency distribution of a piano note obtained from a HRMP analysis. It represents simultaneously structures of very different scales. First $\Gamma$ horizontal lines $\Gamma$ corresponding to large scale atoms $\mathrm{d}$ describe the harmonic structures: the bottom line corresponds to the fundamental frequency of the piano note at $830 \mathrm{~Hz}$ Tand the lines above display the partials; in the bottom left partTshorter horizontal lines display the resonance of the piano's sounding board at $20 \mathrm{~Hz}$. Then vertical features Tcorresponding to fine scale transitory structures $\Gamma$ describe both the attack Tat the beginning of the note (left part) Tand the fall back of the piano's damper on the string $\Gamma$ at its end (bottom right part).

Because of the new correlation function $\Gamma$ the atoms chosen for the decomposition have a smaller time support than with a usual Matching Pursuit decomposition $\Gamma$ hence $\Gamma$ because of Heisenberg inequalities $\Gamma$ they also have a larger frequency support. HRMP thus performs a higher time-resolution decomposition than MPГbut its frequencyresolution is decreased. However for audio applications the most important is to keep a good lo- 


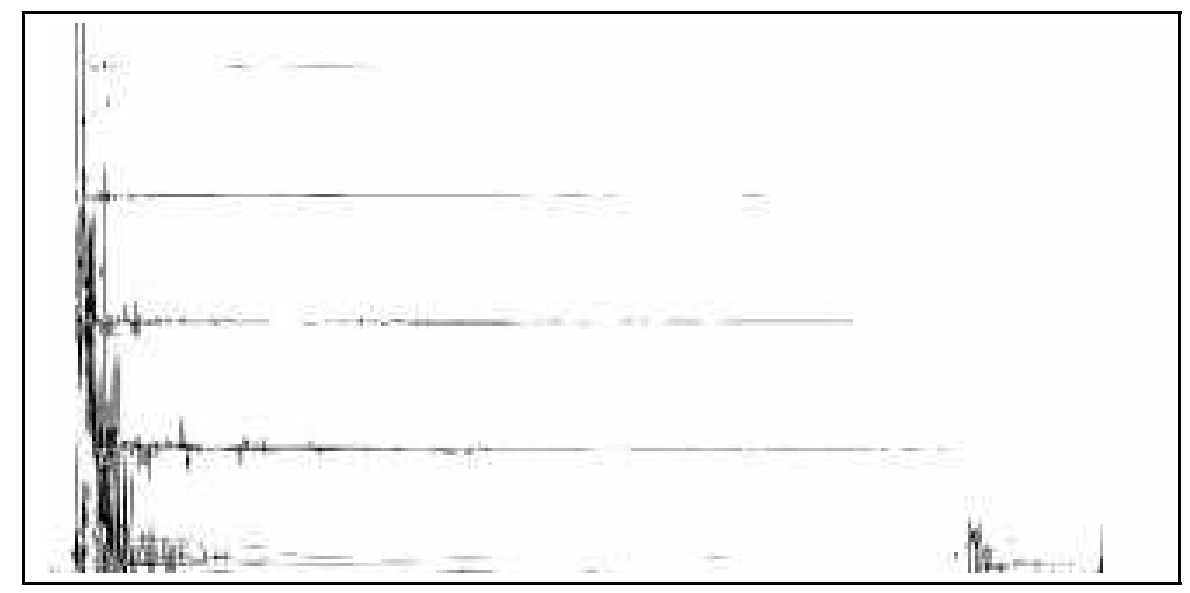

Figure 2: Time-Frequency distribution of a piano note with HRMP

calization of the attacks sensitive to transients : hearing the attack of a musical instrument is almost sufficient to identify it. HRMP is thus adapted to the requirements of sound recording analysis. It is likely to be adapted to many musical applications $\Gamma$ for example to allow the extraction of the parameters of formantwave functions synthesizers [4] $\Gamma$ which could not be achieved with existing analysis processes.

\section{REFERENCES}

[1] S. Mallat and Z. Zhang. Matching pursuit with time-frequency dictionaries. IEEE Trans. Signal Process.Г 41(12):3397-3415Г December 1993.

[2] S. Chen and D.L. Donoho. Atomic decomposition by basis pursuit. Technical report TStatistics DepartmentГStanford UniversityГ 1995.

[3] S. JaggiTW.C. CarlГS. MallatTand A.S. Willsky. High resolution pursuit for feature extraction. Technical reportГMITГNovember 1995.

[4] X. Rodet. Time-domain formant-wave functions synthesis. In Spoken Language Generation and Understanding $\Gamma$ pages 429-441. D. Reidel Publishing CompanyГ1980. 